\title{
ORT_29 - Molecular characterization of optochin - resistant strains of Streptococcus pneumoniae. Implications in laboratory diagnostic
}

Ana Carolina Carvalho de Oliveira ${ }^{*}$; João Fernando Bernardo da $\operatorname{Costa}^{1}$; Ivano de Filippis ${ }^{1}$. ${ }^{1}$ Fiocruz/INCQS.

Introduction: Streptococcus pneumoniae, or pneumococcus, is a Gram positive bacteria, responsible for a large number of pneumonia cases worldwide. Optochin is an antibiotic used for pneumococcus identification in routine laboratories since pneumococci strains are expected to be sensitive to this drug, in addition to being a simple, fast and inexpensive method. Opt acts on the enzyme ATPase, interfering with bacterial metabolism. Studies show that resistant strains to optochin are due to mutations in the subunits of the atpC gene that codes for the ATPase enzyme, target of the antibiotic.

Objective: Quantify strains of $S$. pneumoniae resistant to optochin, deposited in the research collection of INCQS isolated during the pre and post conjugate vaccine introduction. We will also characterize optochin resistant strains in serotypes, MLST and susceptibility to other antimicrobials.

Methodology: Pre and post - vaccine strains were used in this study, comprising the years 2006 to 2020. Susceptibility to optochin was determined by the disk-diffusion method with the most used antimicrobial agents against pneumococci: penicillin, levofloxacin, erythromycin, tetracycline and chloramphenicol. Strains showing resistance to antibiotics after disk-diffusion test are subsequently subjected to the MIC test with e-test strips. Serotypes are determined by serology and confirmed through PCR. Optochin resistant strains showing decreased susceptibility to other antibiotics, will be subjected to sequencing of the atp gene to confirm resistance by modification of target gene and their ST will be determined according to MLST protocol.

Results: Of the 68 strains analyzed so far, 8 showed resistance to optochin and 23 strains showed resistance at least one of the five antibiotics. Only one strain showed resistance to at least one antibiotic from three different classes (ERY, TET e PEN) and was classified as multi-drug resistant (MDR). This strain was sensitive to optochin. Only one strain resistant to optochin was also resistant to TET. The next step will be sequencing the atp gene of optochin resistant strains to determine the mutations that may have lead to the resistant pattern. In addition, we will determine resistance mechanisms for the other antimicrobials.

Conclusion: The results show that resistance to antimicrobial is growing fast. Resistance to optochin, shows the need for constant monitoring and to develop another rapid method. Other analysis to determine serotypes and ST by MLST, will be carried out with the resistant strains to optochin and other antibiotics throughout 2021.

Keywords: Streptococcus pneumoniae; optochin resistance; resistance mechanisms 